https://doi.org/10.15407/ujpe64.6.451

V.YU. HAURYSH, ${ }^{1}$ V.V. ANDREEV ${ }^{2}$

${ }^{1}$ Sukhoi State Technical University of Gomel

(48, Prospect Octyabrya, Gomel 246746, Republic of Belarus; e-mail: mes0n@tut.by)

2 Francisk Skorina Gomel State University

(98, Sovetskaya Str., Gomel 246019, Republic of Belarus; e-mail: vik.andreev@gsu.by)

\title{
DALITZ DECAYS OF UNFLAVORED MESONS IN THE POINCARÉ COVARIANT QUARK MODEL
}

\begin{abstract}
The procedure for obtaining the radiative decay constants of pseudoscalar and vector unflavored mesons in the point-form of PiQM is presented. Based on the obtained earlier parameters of the model for light $(u, d, s)$ quarks, we obtain the remaining parameters from the experiments on the $V \rightarrow P \gamma$ and $P \rightarrow V \gamma$ decays. As a result, the mixing angles for pseudoscalar and vector mesons and the behavior of the $V \rightarrow P \ell^{+} \ell^{-}$decay constant are obtained in agreement with modern experimental data.

Ke ywords: Poincaré, point-form, quark, meson, lepton pair.
\end{abstract}

\section{Introduction}

The problem of description of the decays of hadrons, as bound quark systems, is an important task of high-energy and theoretical physics. Due to several well-known problems of quantum electrodynamics, a number of models of and approaches to this problem were developed. However any of these approaches requires a further modification, since the mechanics of a bound quark interaction has not been completely studied.

The particular importance of researches in this area became obvious, because of the appearance of modern experimental data on lepton decays of light pseudoscalar and vector mesons, as well as on heavy lepton decays (see [1-4]). In view of those and more recent experimental researches, a series of papers were devoted to the calculation of various hadron formfactors of such decays and other observables.

Regarding the decays, which include a hadron in the final state, most convenient for developing the theory of inner quark dynamics are the processes

(C) V.YU. HAURYSH, V.V. ANDREEV, 2019

ISSN 2071-0194. Ukr. J. Phys. 2019. Vol. 64, No. 6 with emitting $\gamma$-quantum $V(P) \rightarrow P(V) \ell^{+} \ell^{-}$and $V(P) \rightarrow P(V) \gamma$. It was found that such decays have the simplest decay mechanism and allow one to find a number of phenomenological characteristics of hadrons such as magnetic moments, different hadron form-factors, mixing angles of quark and gluons, etc. As a consequence, those processes are a more convenient testing ground for any theory describing the quark dynamics, than purely hadronic decays. The former make it possible not only to obtain the behavior of the transition form-factors of mesons, but to estimate the mixing angles for vector and pseudoscalar mesons. This problem is particularly actual due to the latest experimental data on the decays of pseudoscalar $\eta$ and $\eta^{\prime}$-mesons [5]. In addition, the study of mesons within the relativistic quark models gives a reliable description of the hadron formfactors in elastic regions [6], where the resonance and rescattering effects can be neglected. Numerical calculations in such approaches require no dispersive methods [7], which are significant in calculations in the scattering region from $\sim 0.5 \mathrm{GeV}$ and above. 
Though a number of papers with calculations within the instant- and front-forms of the Poincaréinvariant quantum mechanics (PiQM) are available $[8,9]$, the point-form of PiQM, due to several difficulties, is rarely used for these purposes. Therefore, a further development of this form of the dynamics is an actual problem of hadron physics.

The paper is organized as follows. We consider the model parameters based on the point-form of PiQM without any use of the explicit form of the interquark potential in Section 2. As a particularity of the calculations, we mention the usage of integral representations for the decay of pseudoscalar and vector mesons in a leptonic pair, as well as the pseudoscalar density, to estimate the values of constituent quark masses and the parameters of wave functions. In Section 3, the anomalous magnetic moments of quarks of the light sector are numerically evaluated, by basing on the obtained earlier expression for the channel $V \rightarrow P \gamma$. In Section 4, the comparative analysis of the obtained results on the constituent quark masses and the anomalous magnetic moments of quarks of the light sector is carried out. In section 5 , the behavior of the form-factors for neutral $\phi$ and $\omega$ mesons is studied and compared with present-day experimental data.

\section{Parameters of the Model Based on the Point-Form of PiQM}

Here, we introduce the basic parameters obtained earlier in [10-12]. Let us briefly discuss the basic steps of the calculation scheme. By using the relation for the state vector of a meson with momentum $\mathbf{Q}$, angular momentum $J$, and mass $M$ in the point-form of PiQM

$$
\begin{aligned}
& |\mathbf{Q}, J \mu, M\rangle=\sum_{\lambda_{1}, \lambda_{2}} \sum_{\nu_{1}, \nu_{2}} \int \mathrm{d} \mathbf{k} \Phi_{\ell S}^{J}\left(\mathrm{k}, \beta_{q \bar{Q}}^{I}\right) \times \\
& \times \sqrt{\frac{\omega_{m_{q}}\left(\mathrm{p}_{1}\right) \omega_{m_{\bar{Q}}}\left(\mathrm{p}_{2}\right) M_{0}(\mathrm{k})}{\omega_{m_{q}}(\mathrm{k}) \omega_{m_{\bar{Q}}}(\mathrm{k}) \omega_{M_{0}}\left(\mathrm{P}_{12}\right)}} \Omega\left\{\begin{array}{c}
\ell \underset{j}{\ell} J \\
\nu_{1}, \nu_{2}, \mu
\end{array}\right\}\left(\theta_{k}, \phi_{k}\right) \times \\
& \times D_{\lambda_{1}, \nu_{1}}^{1 / 2}\left(\mathbf{n}_{W_{1}}\right) D_{\lambda_{2}, \nu_{2}}^{1 / 2}\left(\mathbf{n}_{W_{2}}\right)\left|\mathbf{p}_{\mathbf{1}}, \lambda_{1}, \mathbf{p}_{\mathbf{2}}, \lambda_{2}\right\rangle
\end{aligned}
$$

we can obtain the integral representation of pseudoscalar and vector mesons $f_{P, V}\left(q, \bar{Q}, \beta_{q \bar{Q}}^{P, V}\right)$, as well as the pseudoscalar density constant $g\left(q, \bar{Q}, \beta_{q \bar{Q}}^{P}\right)$ [10]. After some calculations with the oscillator wave function for pseudoscalar $(I=P, \ell=0)$ and vector mesons $(I=V, \ell=0)$

$\Phi\left(\mathrm{k}, \beta_{q \bar{Q}}^{I}\right)=\frac{2}{\pi^{1 / 4}\left(\beta_{q \bar{Q}}^{V, P}\right)^{3 / 2}} \exp \left[-\frac{\mathrm{k}^{2}}{2\left(\beta_{q \bar{Q}}^{I}\right)^{2}}\right]$,

the integral representation with regard for the current quark masses yields the following parameters of the model under study [12]:

$m_{u}=(219.48 \pm 9.69) \mathrm{MeV}$,

$m_{d}=(221.97 \pm 9.69) \mathrm{MeV}$,

$m_{s}=(416.95 \pm 61.22) \mathrm{MeV}$,

$\beta_{u \bar{d}}^{P_{\bar{d}}}=(367.93 \pm 25.10) \mathrm{MeV}$,

$\beta_{u \bar{d}}^{V}=(311.95 \pm 2.14) \mathrm{MeV}$,

$\beta_{u \bar{s}}^{P}=(375.53 \pm 19.66) \mathrm{MeV}$,

$\beta_{u \bar{s}}^{V}=(313.62 \pm 24.22) \mathrm{MeV}$.

For the further calculations with the use of a weak isotopic symmetry violation, we assume that

$\beta_{u \bar{u}}^{V}=\beta_{u \bar{d}}^{V}+\triangle \beta_{u \bar{d}}, \beta_{d \bar{d}}^{V}=\beta_{u \bar{d}}^{V}-\triangle \beta_{u \bar{d}}$,

$\beta_{d \bar{s}}^{V}=\beta_{u \bar{s}}^{V}-\triangle \beta_{u \bar{d}}, \beta_{d \bar{s}}^{P}=\beta_{u \bar{s}}^{P}+\triangle \beta_{u \bar{d}}$,

where $\triangle \beta_{u \bar{d}} \simeq m_{d}-m_{u}=(2.5 \pm 0.2) \mathrm{MeV}$.

\section{Radiative Decay Constant in PiQM}

The parametrization of the matrix element for the transition of a vector (pseudoscalar) meson $V(P)$ into a pseudoscalar (vector) meson $P(V)$ with 4-momenta $Q=\left\{Q_{0}, \mathbf{Q}\right\}\left(Q^{2}=M^{2}\right)$ and $Q^{\prime}=\left\{Q_{0}^{\prime}, \mathbf{Q}^{\prime}\right\}\left(Q^{\prime 2}=\right.$ $\left.=M^{\prime 2}\right)$ with the emission of a virtual $\gamma^{*}$ is given by $[13,14]$

$$
\begin{aligned}
& \left\langle\mathbf{Q}^{\prime}, M^{\prime}\left|\hat{J}_{h}^{\mu}(0)\right| \mathbf{Q}, M\right\rangle=i \sqrt{4 \pi \alpha} g_{V P \gamma^{*}}\left(q^{2}\right) \times \\
& \times \frac{\epsilon^{\mu \nu \rho \sigma} \varepsilon_{\nu}\left(\lambda_{V}\right) Q_{\rho} Q_{\sigma}}{(2 \pi)^{3} \sqrt{4 Q_{0} Q_{0}^{\prime}}}
\end{aligned}
$$

where $\varepsilon_{\nu}\left(\lambda_{V}\right)$ is the polarization vector of a meson $V$, and $\alpha=e^{2} / 4 \pi$ is the fine structure constant. In the proposed approach, the decay constant of $V(P) \rightarrow$ $\rightarrow P(V) \gamma^{*}$ from (3.1) is defined by

$$
\begin{aligned}
& g_{V P \gamma^{*}}\left(q^{2}\right)=(2 \pi)^{3} \frac{\sqrt{4 V_{0}^{\prime} V_{0}}}{\sqrt{M_{0}\left(\mathrm{k}^{\prime}\right) M_{0}(\mathrm{k})}} \times \\
& \times\left\langle\mathbf{Q}^{\prime}, M^{\prime}\left|\frac{\left(\hat{J}_{h}(0) K^{*}\left(\lambda_{V}\right)\right)}{\left(K\left(\lambda_{V}\right) K^{*}\left(\lambda_{V}\right)\right)}\right| \mathbf{Q}, 1 \lambda_{V}, M\right\rangle,
\end{aligned}
$$

where $K^{\mu}\left(\lambda_{V}\right)=i \epsilon^{\mu \nu \rho \sigma} \varepsilon_{\nu}\left(\lambda_{V}\right) V_{\rho} V_{\sigma}^{\prime}$. Assume that the studied decay is caused by the interaction of 
constituent quarks, which are included in the meson $V(P)$. For the virtual $\gamma$-quantum, we have the vertex

$\Gamma_{q, \bar{Q}}^{\mu}=F_{1}\left(q^{2}\right) \gamma^{\mu}+\frac{1}{2 m_{q, \bar{Q}}} F_{2}\left(q^{2}\right) \sigma^{\mu \nu} q_{\nu}$,

$t=q^{2}=\left(Q-Q^{\prime}\right)^{2}$

where

$F_{1}(0)+F_{2}(0)=\mu_{q, \bar{Q}}, \mu_{q, \bar{Q}}=\frac{e_{q, \bar{Q}}}{2 m_{q, \bar{Q}}}\left(1+\kappa_{q, \bar{Q}}\right)$

in the generalized Breit system $\left(\mathbf{V}_{Q}+\mathbf{V}_{Q^{\prime}}=0\right)$. In the limit $q^{2} \rightarrow 0$, we can obtain the integral representation of the radiative decay constant for $V \rightarrow P \gamma$ decay [12]:

$g_{V P \gamma}=\int \mathrm{dk} \mathrm{k}{ }^{2} \Phi\left(\mathrm{k}, \beta_{q \bar{Q}}^{V}\right) \Phi^{*}\left(\mathrm{k}, \beta_{q \bar{Q}}^{P}\right) \times$

$\times\left(e_{q} f_{1}\left(\mathrm{k}, m_{q}, m_{\bar{Q}}\right)+\frac{e_{q} \kappa_{q}}{2 m_{q}} f_{2}\left(\mathrm{k}, m_{q}, m_{\bar{Q}}\right)-\right.$

$\left.-e_{\bar{Q}} f_{1}\left(\mathrm{k}, m_{\bar{Q}}, m_{q}\right)-\frac{e_{\bar{Q}} \kappa_{\bar{Q}}}{2 m_{\bar{Q}}} f_{2}\left(\mathrm{k}, m_{\bar{Q}}, m_{q}\right)\right)$,

where

$f_{1}\left(\mathrm{k}, m_{q}, m_{\bar{Q}}\right)=$

$\frac{1}{3 \omega_{m_{q}}(\mathrm{k})}\left(\frac{m_{q}+m_{\bar{Q}}}{M_{0}(\mathrm{k})}+\frac{m_{q}}{\omega_{m_{q}}(\mathrm{k})}+1\right)$,

$f_{2}\left(\mathrm{k}, m_{q}, m_{\bar{Q}}\right)=-\frac{2}{3}\left(\frac{m_{q}}{\omega_{m_{q}}(\mathrm{k})}+\frac{m_{q}^{2}}{\omega_{m_{q}}^{2}(\mathrm{k})}+1\right)$

and

$$
\begin{aligned}
& M_{0}(\mathrm{k})=\omega_{m_{q}}(\mathrm{k})+\omega_{m_{\bar{Q}}}(\mathrm{k}), \\
& \omega_{m}(\mathrm{k})=\sqrt{\mathrm{k}^{2}+m^{2}}, \mathrm{k}=|\mathbf{k}| .
\end{aligned}
$$

The values of the magnetic moments of quarks ( $u$, $d$, and $s$ ) can be found from the matching of the results of theoretical calculations with experimental data [1]. Using the $\rho^{+}, K^{*+}$ and $K^{* 0}$ decay values from (3.6), we get $\kappa_{u}=(-0.123 \pm 0.084), \kappa_{d}=$ $=(-0.088 \pm 0.015)$, and $\kappa_{s}=(-0.198 \pm 0.011)$ (we use natural quark units). The analysis shows that the anomalous magnetic moments in our work are in good agreement with other models and assumptions. In view of the values $\tilde{\kappa}_{u}=-0.064$ and $\tilde{\kappa}_{d}=0.017$ in [15], we can get $\tilde{\kappa}_{u}=e_{u} \kappa_{u}=-0.082, \tilde{\kappa}_{d}=e_{d} \kappa_{d}=$ $=0.029$.

\section{Mixing Scheme of Mesons}

Due to various mixing effects, the mesons are usually a linear combinations of state vectors (2.1), which differ from each other by their quark composition. So, in this section, we briefly discuss the mixing scheme scenarios for the unflavored pseudoscalar and vector mesons.

The following mixing schemes are widely used in physical applications [16]:

$\left\{\begin{array}{l}\psi_{1}=(1 / \sqrt{2})|u \bar{u}-d \bar{d}\rangle \\ \psi_{q}=(1 / \sqrt{2})|u \bar{u}+d \bar{d}\rangle \\ \psi_{s}=|s \bar{s}\rangle\end{array}\right.$

and

$\left\{\begin{array}{l}\psi_{1}=(1 / \sqrt{2})|u \bar{u}-d \bar{d}\rangle \\ \psi_{8}=(1 / \sqrt{6})|u \bar{u}+d \bar{d}-2 s \bar{s}\rangle \\ \psi_{0}=(1 / \sqrt{3})|u \bar{u}+d \bar{d}+s \bar{s}\rangle\end{array}\right.$

By means of (4.1 and 4.2), the physical states of vector mesons are defined by

$\left(\begin{array}{c}|\phi\rangle \\ \omega\rangle \\ \left|\rho^{0}\right\rangle\end{array}\right)=U\left(\phi_{V}, \phi_{\rho \phi}, \phi_{\rho \omega}\right)\left(\begin{array}{c}\psi_{q} \\ \psi_{s} \\ \psi_{1}\end{array}\right)$

or

$\left(\begin{array}{c}|\phi\rangle \\ \omega\rangle \\ \left|\rho^{0}\right\rangle\end{array}\right)=U\left(\theta_{V}, \theta_{\rho \phi}, \theta_{\rho \omega}\right)\left(\begin{array}{c}\psi_{8} \\ \psi_{0} \\ \psi_{1}\end{array}\right)$

where $U(\alpha, \beta, \gamma)$ is a rotation matrix.

It is known that the mixing angle $\phi_{\rho \omega}$ is due to QCD processes. It is a complex number, whose numerical value is known with large error. So, we assume that $\phi_{\rho \omega}=0$; the mixing angle $\rho-\phi$ also can be neglected. In the final analysis, we will use only one $\phi_{V}$-angle [20] for vector mesons. For it, relation (4.2) implies that

$\left\{\begin{array}{l}|\phi\rangle=\cos \phi_{V} \psi_{q}-\sin \phi_{V} \psi_{s} \\ |\omega\rangle=\sin \phi_{V} \psi_{q}+\cos \phi_{V} \psi_{s} \\ \left|\rho^{0}\right\rangle=\psi_{1}\end{array}\right.$

where the $\phi_{V}$-angle is related to the $\theta_{V}$-angle [16] by $\theta_{V}=\phi_{V}-\arctan \sqrt{2}$.

For the pseudoscalar sector, respectively, we have

$\left(\begin{array}{c}|\eta\rangle \\ \left|\eta^{\rangle}\right\rangle \\ \left|\pi^{0}\right\rangle\end{array}\right)=U\left(\phi_{P}, \phi_{\pi \eta}, \phi_{\pi \eta^{\prime}}\right)\left(\begin{array}{c}\psi_{q} \\ \psi_{s} \\ \psi_{1}\end{array}\right)$

ISSN 2071-0194. Ukr. J. Phys. 2019. Vol. 64, No. 6 
where the mixing effects for $\pi^{0}-\eta$ and $\pi^{0}-\eta^{\prime}$ are extremely small. The naive mixing scheme (4.5) leads to

$\left\{\begin{array}{l}|\eta\rangle=\cos \phi_{P} \psi_{q}-\sin \phi_{P} \psi_{s} \\ \left|\eta^{\prime}\right\rangle=\sin \phi_{P} \psi_{q}+\cos \phi_{P} \psi_{s} \\ \left|\pi^{0}\right\rangle=\psi_{1}\end{array}\right.$

However, the analysis of the decay $\eta-\eta^{\prime}$ shows that scheme (4.6) with one mixing angle is not sufficient for describing the experimental data. Therefore, the number of independent mixing parameters $\eta-\eta^{\prime}$ was increased due to the gluonium content [5]. As a result, the physical states for pseudoscalar mesons could be written as [17]

$$
\left(\begin{array}{c}
|\eta\rangle \\
\left|\eta^{\prime}\right\rangle \\
G\rangle
\end{array}\right)=U\left(\phi_{P}, \alpha_{G}, \phi_{G}\right)\left(\begin{array}{c}
\psi_{q} \\
\psi_{s} \\
\psi_{G}
\end{array}\right)
$$

or, using the explicit form of the $U\left(\phi_{P}, \alpha_{G}, \phi_{G}\right)$ matrix,

$\left\{\begin{array}{l}|\eta\rangle=X_{\eta} \psi_{q}+Y_{\eta} \psi_{s}+Z_{\eta} \psi_{G}, \\ \left|\eta^{\prime}\right\rangle=X_{\eta^{\prime}} \psi_{q}+Y_{\eta^{\prime}} \psi_{s}+Z_{\eta^{\prime}} \psi_{G}, \\ |G\rangle=X_{G} \psi_{q}+Y_{G} \psi_{s}+Z_{G} \psi_{G},\end{array}\right.$

where $|G\rangle$ is the gluonium content, and

$X_{\eta}=\cos \phi_{P} \cos \alpha_{G}, Y_{\eta}=-\sin \phi_{P} \cos \alpha_{G}$,

$Z_{\eta}=-\sin \alpha_{G}, Z_{\eta^{\prime}}=\cos \alpha_{G} \sin \phi_{G}$,

$X_{\eta^{\prime}}=\cos \phi_{P} \sin \alpha_{G} \sin \phi_{G}+\sin \phi_{P} \cos \phi_{G}$,

$Y_{\eta^{\prime}}=\cos \phi_{P} \cos \phi_{G}-\sin \phi_{P} \sin \alpha_{G} \sin \phi_{G}$.

Using the obtained model parameters and quark magnetic moments (see Sec. 2 and 3) and accounting

Comparison of experimental

$\Gamma^{(\text {exp. })}$ and theoretical values $\Gamma^{(\text {th. })}$

for the $V(P) \rightarrow P(V) \gamma$-decay with regard

for anomalous quark magnetic moments

\begin{tabular}{|c|c|c|}
\hline Radiative decay & $\Gamma^{\text {(exp.) }, \mathrm{keV}}$ & $\Gamma^{(\text {th. })}, \mathrm{keV}$ \\
\hline$\omega \rightarrow \pi^{0} \gamma$ & $687 \pm 19[5]$ & $704 \pm 13$ \\
& $714 \pm 20[1]$ & \\
$\omega \rightarrow \eta \gamma$ & $5.8 \pm 1.1[1]$ & $6.8 \pm 2.3$ \\
$\phi \rightarrow \pi^{0} \gamma$ & $5.5 \pm 0.2[1]$ & $5.6 \pm 2.1$ \\
$\phi \rightarrow \eta \gamma$ & $55.4 \pm 1.2[1]$ & $55.7 \pm 2.5$ \\
$\phi \rightarrow \eta^{\prime} \gamma$ & $0.27 \pm 0.01[1]$ & $0.27 \pm 0.14$ \\
$\rho^{0} \rightarrow \pi^{0} \gamma$ & $77 \pm 28[18]$ & $83 \pm 16$ \\
$\rho^{0} \rightarrow \eta \gamma$ & $44.7 \pm 3.2[1]$ & $44.8 \pm 3.5$ \\
$\eta^{\prime} \rightarrow \rho^{0} \gamma$ & $56.7 \pm 2.7[1]$ & $56.9 \pm 2.3$ \\
$\eta^{\prime} \rightarrow \omega \gamma$ & $5.1 \pm 0.4[1]$ & $5.1 \pm 0.8$ \\
\hline
\end{tabular}

for that the standard model gives no evidence for the $G-\gamma-G$ vertex, we match experimental data with the model calculations in the table.

In the calculations, we used

$\beta_{u \bar{u}}^{P}=(280.60 \pm 25.07) \mathrm{MeV}$,

$\beta_{d \bar{d}}^{P}=(277.95 \pm 25.07) \mathrm{MeV}$,

$\beta_{s \bar{s}}^{P}=(494.54 \pm 19.66) \mathrm{MeV}$,

$\theta_{P}=(-9.1 \pm 2.4)^{\circ}$,

and the mixing angle for the meson sector $\theta_{V}=$ $=(31.92 \pm 0.2)^{\circ}[19]$.

\section{Form-Factors of the $V \rightarrow P \ell^{+} \ell^{-}$ Decay in the Point-Form of PiQM}

The methods of obtaining the decay constant $V(P) \rightarrow P(V) \gamma^{*}$ is presented in [12]. The final expression in the case of the equal quark masses $m_{\bar{Q}}=m_{\bar{q}}$ takes the form:

$g_{V P \gamma^{*}}\left(q^{2}=t\right)=\frac{1}{4 \pi} \sum_{\nu_{1}, \nu_{1}^{\prime}} \int \mathrm{d} \mathbf{k} \sqrt{\frac{3+4 \nu_{1}\left(\lambda_{V}-\nu_{1}\right)}{4}} \times$

$\times \frac{\nu_{1}^{\prime}}{\omega_{m_{q}}(\mathrm{k}) \sqrt{M_{0}(\mathrm{k})}} \Phi\left(\mathrm{k}, \beta_{q \bar{q}}^{V}\right)\left(\frac{1}{\sqrt{\varpi^{2}(\mathrm{k}, t)-1}} \times\right.$

$\times \frac{\Phi^{*}\left(\mathrm{k}_{2}, \beta_{q \bar{q}}^{P}\right)}{\sqrt{M_{0}\left(\mathrm{k}_{2}\right)}} D_{-\nu_{1}^{\prime}, \lambda_{V}-\nu_{1}}\left(\mathbf{n}_{W_{2}}\left(\mathbf{k}, \boldsymbol{v}_{Q}\right)\right) \bar{u}_{\nu_{1}^{\prime}}\left(\mathbf{k}_{2}, m_{q}\right) \times$

$\times B\left(\boldsymbol{v}_{Q}\right)\left(K^{*}\left(\lambda_{V}\right) \cdot \Gamma_{q}\right) u_{\nu_{1}}\left(\mathbf{k}, m_{q}\right)+\frac{1}{\sqrt{\varpi^{2}(\mathrm{k}, t)-1}} \times$ $\times \frac{\Phi^{*}\left(\mathrm{k}_{1}, \beta_{q \bar{q}}^{P}\right)}{\sqrt{M_{0}\left(\mathrm{k}_{1}\right)}} D_{\nu_{1}^{\prime}, \nu_{1}}\left(\mathbf{n}_{W_{1}}\left(\mathbf{k}, \boldsymbol{v}_{Q}\right)\right) \bar{v}_{\lambda_{V}-\nu_{1}}\left(\mathbf{k}, m_{\bar{q}}\right) \times$ $\left.\times B\left(-\boldsymbol{v}_{Q}\right)\left(K^{*}\left(\lambda_{V}\right) \Gamma_{\bar{q}}\right) v_{-\nu_{1}^{\prime}}\left(\mathbf{k}_{1}, m_{\bar{q}}\right)\right)$.

In expression (5.1) in the Breit system, [12]

$\boldsymbol{v}_{Q}=\frac{\mathbf{V}_{Q}}{V_{0}}$

$K\left(\lambda_{V}\right)=\sqrt{\frac{\varpi^{2}(\mathrm{k}, t)-1}{2}} \times\left\{0, \lambda_{V}, i, 0\right\}$,

$\varpi^{2}(\mathrm{k}, t)=1-\frac{t}{4\left(\omega_{m_{q}}^{2}(\mathrm{k})-\mathrm{k}^{2} \cos ^{2} \theta_{\mathrm{k}}\right)}$

and

$\mathbf{k}_{1,2}=\mathbf{k} \pm \boldsymbol{v}_{Q}\left((\varpi(\mathrm{k}, t)+1) \omega_{m_{q, \bar{q}}}(\mathrm{k})-\right.$

$\left.-\mathrm{k} \sqrt{\varpi^{2}(\mathrm{k}, t)-1} \cos \theta_{\mathrm{k}}\right)$.

ISSN 2071-0194. Ukr. J. Phys. 2019. Vol. 64, No. 6 


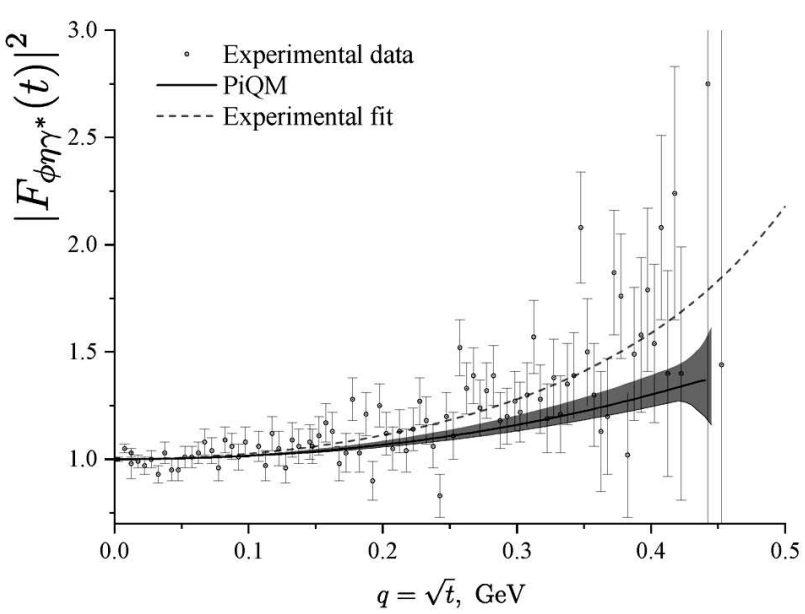

Fig. 1. Behavior of the form-factor of the decay $\phi \rightarrow \rightarrow \eta \ell^{+} \ell^{-}$ for different $q$ values. The experimental data with the fitting parameter $b_{\phi \eta}=(1.3 \pm 0.1) \mathrm{GeV}^{-2}$ are taken from [2]

Fixing the vertex of the decay with expression (3.3), where [15]

$$
\begin{aligned}
F_{1}(t) & =\frac{e_{q}}{1-\frac{\left\langle r_{q}\right\rangle^{2}}{6} t}, F_{2}(t)=\frac{e_{q} \kappa_{q}}{\left(1-\frac{\left\langle r_{q}\right\rangle^{2}}{12} t\right)^{2}}, \\
\left\langle r_{q}\right\rangle^{2} & =\frac{a}{m_{q}^{2}},
\end{aligned}
$$

it becomes possible to carry out the research of the behavior of the form-factors of the meson as functions of the momentum transferred to the leptonic pair.

We will study the Dalitz decays $V \rightarrow P \ell^{+} \ell^{-}$ with the parameter $a=0.3[15]$ (we assume that, for an $s$-quark, the parameter is the same as for $u$ and $d$-quarks [21]). Using representations (4.5) and (4.10) of the state vectors for vector and pseudoscalar mesons, respectively, with regard for the orthogonality of the vectors with different quark flavors, we obtain the behavior of the form-factor $F_{V P \gamma^{*}}(t)=$ $=g_{V P \gamma^{*}}(t) / g_{V P \gamma}$. For the decays $\phi \rightarrow \eta \ell^{+} \ell^{-}, \phi \rightarrow$ $\rightarrow \pi^{0} \ell^{+} \ell^{-}$, and $\omega \rightarrow \pi^{0} \ell^{+} \ell^{-}$presented in Figs. $1-3$.

We will make analysis of the obtained results given in in Figs. 1-3. It is obvious that the calculations in the context of the suggested model within the limits of errors correspond to the experimental behavior of the electromagnetic form-factors of $\phi$ and $\omega$ mesons in the interval $q<0.5 \mathrm{GeV}$.

As for the obtained value of the mixing $\theta_{P}$-angle (see 4.12), it is well known the recent tendency to take the gluonium content into account leads to the results $\theta_{P} \in\left(-20^{\circ} ;-10^{\circ}\right)$ : in [16], the value $\theta_{P} \approx-17^{\circ}$

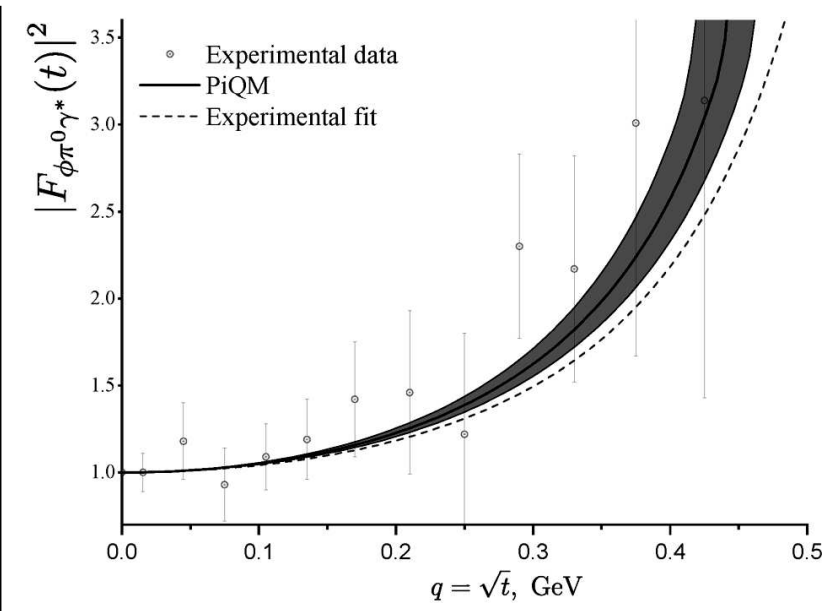

Fig. 2. Behavior of the form-factor of the decay $\phi \rightarrow \rightarrow$ $\pi^{0} \ell^{+} \ell^{-}$for different $q$ values. The experimental data with the fitting parameter $b_{\phi \pi^{0}}=(2.0 \pm 0.1) \mathrm{GeV}^{-2}$ are taken from [3]

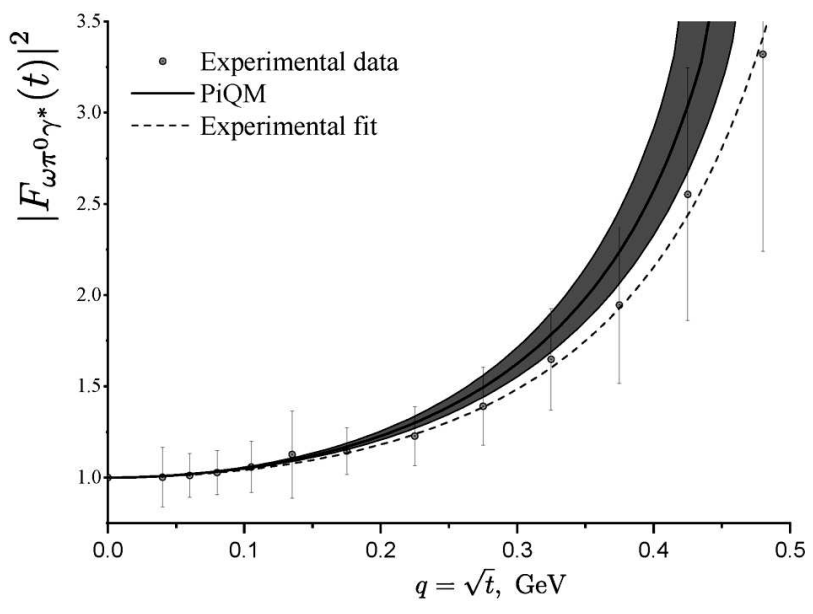

Fig. 3. Behavior of the form-factor of the decay $\omega \rightarrow \pi^{0} \ell^{+} \ell^{-}$ for different $q$ values. The experimental data with the fitting parameter $b_{\omega \pi^{0}}=(1.9 \pm 0.2) \mathrm{GeV}^{-2}$ are taken from [4]

was used for $\mathrm{SU}(3)$-breaking effects; the hidden-localsymmetry model gave $\theta_{P} \approx-11^{\circ}$ [23], while the analysis of the experimental data yields $\theta_{P} \approx-12^{\circ}$ [5]. Thus, the problem of fixing the $\theta_{P}$-angle of the pseudoscalar sector has no exact solution. The value $\theta_{P}=(-9.1 \pm 2.4)^{\circ}$ obtained in our work is close, within reasonable limits, to other approaches and models.

\section{Conclusion}

We have carried out the research of the behavior of the form-factors of light neutral vector mesons for dif- 
ferent momenta transferred to the leptonic pair with regard for anomalous magnetic quark moments. We used the parameters that were obtained from the leptonic decays of pseudoscalar and vector mesons. The analysis of the obtained results demonstrates that the suggested self-consistent model based on the pointform of Poincaré-invariant quantum mechanics describes adequately both leptonic and hadronic decays of pseudoscalar and vector mesons.

1. M. Tanabashi et al. (Particle data group). Review of particle physics. Phys. Rev. D 98, 030001 (2018).

2. D. Babusci et al. (KLOE Collaboration). Study of the dalitz decay $\phi \rightarrow \eta e^{+} e^{-}$with the KLOE detector. Phys. Lett. B 742, 1 (2015).

3. A. Anastasi et al. (KLOE Collaboration). Measurement of the $\phi \rightarrow \pi^{0} e^{+} e^{-}$transition form factor with the KLOE detector. Phys. Lett. B 757, 362 (2015).

4. P. Adlarson et al. (A2 Collaboration). Measurement of the $\omega \rightarrow \pi^{0} e^{+} e^{-}$and $\eta \rightarrow e^{+} e^{-} \gamma$ Dalitz decays with the A2. Phys. Rev. C 95, 035208 (2017).

5. F. Ambrosino et al. (KLOE Collaboration). $\eta, \eta$ physics at KLOE. PoS CD 09, 045 (2009).

6. I. Caprini. Testing the consistency of the $\omega-\pi$ transition form factor with unitarity and analyticity. Phys. Rev. D 92, 014014 (2015).

7. I. V. Danilkin, C. Fernández-Ramírez, P. Guo, V. Mathieu, D. Schott, M. Shi, A.P. Szczepaniak. Dispersive analysis of $\omega / \phi \rightarrow 3 \pi, \pi \gamma^{*}$. Phys. Rev. D 91, 094029 (2015).

8. W.N. Polyzou, Y. Huang, Ch. Elster. Mini review of Poincaré invariant quantum theory. Few Body Syst. 49, 129 (2011).

9. B.D. Keister, W.N. Polyzou. Relativistic Hamiltonian dynamics in nuclear and particle physics. Adv. Nucl. Phys. 20, 225 (1991).

10. V.V. Andreev, V.Yu. Haurysh. Constituent quark masses in Poincaré-invariant quantum mechanics. J. Phys. Conf. Ser. 938, 012030 (2017).

11. V.V. Andreev, V.Yu. Haurysh. Poincaré-covariant quark model of electroweak light mesons decays. EPJ Web Conf. 204, 08006 (2019).

12. V.V. Andreev, V.Yu. Haurysh. Electroweak decays of unflavored mesons in Poincaré-covariant quark model. Turkish J. of Phys. 43 (2), 167 (2019).
13. W. Jaus. Relativistic constituent quark model of electroweak properties of light mesons. Phys. Rev. D 44, 2851 (1991).

14. W. Jaus. Consistent treatment of spin 1 mesons in the light front quark model. Phys. Rev. D 67, 094010 (2003).

15. R. Petronzio, S. Simula, G. Ricco. Possible evidence of extended objects inside the proton. Phys. Rev. D 67, 094004 (2003).

16. A. Bramon, R. Escribano, M.D. Scadron. Radiative $V \rightarrow$ $\rightarrow P \gamma$ transitions and $\eta-\eta^{\prime}$-mixing. Phys. Let. $B \mathbf{5 0 3}$, 271 (2001).

17. C.E. Thomas. Composition of the pseudoscalar $\eta$ and $\eta^{\prime}$ mesons. JHEP 10, 026 (2007).

18. M.N. Achasov et al. (SND Collaboration). Study of the reaction $e^{+} e^{-} \rightarrow \pi^{0} \gamma$ with the SND detector at the VEPP2M collider. Phys. Rev. D 93, 092001 (2016).

19. G. Amelino-Camelia et al. (KLOE Collaboration). Physics with the KLOE-2 experiment at the upgraded DA $\phi \mathrm{NE}$. Eur. Phys. J. C 68, 619 (2010).

20. W. Qian, B.Q. Ma. Vector meson $\omega-\phi$ mixing and their form factors in the light-cone quark model. Phys. Rev. D 78, 074002 (2008).

21. F. Cardarelli, I.L. Grach, I.M. Narodetsky, E. Pace, G. Salme, S. Simula. Charge form-factor of $\pi$ and $K$ mesons. Phys. Rev. D 53, 6682 (1996).

22. A. Bramon, R. Escribano, M.D. Scadron. The $\eta-\eta^{\prime}$ mixing angle revisited. Eur. Phys. J. C 7, 271 (1999).

23. M. Benayoun, L. DelBuono, S. Eidelman, V.N. Ivanchenko, H.B. O'Connell. Radiative decays, nonet symmetry and SU(3) breaking. Phys. Rev. D 59, 114027 (1999).

Received 07.06.19

В.Ю. Гавриш, В.В.Андреєе

РОЗПАДИ ДАЛІЦА НЕЙТРАЛЬНИХ МЕЗОНІВ

У ПУАНКАРЕ-ІНВАРІАНТНІЙ КВАРКОВІЙ МОДЕЛІ

$\mathrm{P}$ е $з$ ю м е

Представлена процедура отримання констант радіаційного розпаду псевдоскалярних і векторних нейтральних мезонів у точковій формі ПІКМ. В ході роботи автори, виходячи з отриманих раніше параметрів моделі для легких $(u, d, s)$ кварків, отримують решту параметрів з експериментів по $V \rightarrow P \gamma$ і $P \rightarrow V \gamma$ розпадах. В результаті отримано кути змішування для псевдоскалярного і векторного мезонів і поведінку константи розпаду $V \rightarrow P \ell^{+} \ell^{-}$, проведено їх порівняння з сучасними експериментальними даними. 